\title{
Neutron and synchrotron radiation diffraction study of the matrix residual stress evolution with plastic deformation in aluminum alloys and composites
}

\author{
Pedro Fernández-Castrillo ${ }^{1,2}$, Giovanni Bruno ${ }^{1,3+}$, Gaspar González-Doncel ${ }^{2^{*}}$ \\ ${ }^{1}$ Institut Laue-Langevin, ILL, Rue Jules Horowitz, BP 156F-38042 Grenoble Cedex 9, France \\ 2 Dept. of Physical Metallurgy,Centro Nacional de Investigaciones Metalúrgicas (CENIM), \\ C.S.I.C., Av. de Gregorio del Amo 8, E-28040 Madrid, Spain \\ ${ }^{3}$ Manchester Materials Science Centre, Grosvenor Str., Manchester M1 7HS, UK \\ + Present Address, Corning SAS- CETC 7bis, Av. de Valvins, BP 3 , F-77211 Avon, France.
}

\begin{abstract}
The evolution of the matrix residual stress, RS, with compressive and tensile plastic deformation in aluminum alloys and corresponding composites reinforced by alumina particles (15\% in vol.) has been investigated. High and moderate strength aluminum alloys, 2014Al and 6061Al, respectively, have been studied. The RS was determined by neutron and synchrotron radiation diffraction in samples treated to a T6 condition and deformed from 0 to $15 \%$ plastic strain. The results show that compressive plastic deformation relaxes the hydrostatic matrix RS of all materials to a minimum at around $2-5 \%$ plastic strain. At higher strains the hydrostatic matrix RS increases noticeably in the high strength 2014Al alloy and the corresponding composite but only moderately in the 6061 Al alloy and the respective composite. On the other hand, no increase of the RS takes place after a large tensile strain. This difference can be attributed to the higher inhomogeneity of the plastic flow in the high strength matrix materials in compression test.
\end{abstract}

keywords: Aluminum Alloys; Metal Matrix Composites (MMCs); Residual Stress; Plastic Deformation; Neutron diffraction; Synchrotron Radiation Diffraction.

* Corresponding author: Tel.:+34-915538900, ext.337, fax: +34-915347425,

E-mail: ggd@cenim.csic.es 


\section{Introduction}

Metal matrix composites, MMCs, in particular aluminum alloy matrix composites, are structural materials of great interest in the transportation sector. This is due to the improved mechanical properties of these materials (higher stiffness and strength) with respect to the unreinforced alloys [1,2]. The addition of the reinforcement for the improved mechanical properties does not increase significantly the density of the material. Several factors are responsible for these improvements. Among these, the presence of a residual stress, RS, plays an important role. Knowledge of the level of this RS is crucial to optimize performance of composite materials. Although it is well known that RS in materials is strongly dependent on the thermo-mechanical processing, there is not yet enough knowledge on the specific effect of these processes. This is particularly important in MMCs: Besides the common macroscopic RS, M-RS, originated during material and component manufacture, also a microscopic RS, m-RS, is developed. This m-RS is associated to composite microstructure and originated from the different thermal contraction and elasto-plastic behavior of matrix and reinforcement $[3,4]$.

There are several investigations in which the effect of plastic deformation on the RS state has been examined [4-8]. In particular, it has been shown in [8] that the hydrostatic M-RS of several powder metallurgy, PM, 6061Al matrix composites decreases rapidly with compressive plastic deformation until a strain $2-5 \%$ is reached. With further deformation, the M-RS increases. The hydrostatic microscopic RS, m-RS, on the other hand, decreases progressively with large compressive deformation. The increase of the M-RS was attributed to inhomogeneous plastic deformation of composite samples when large compressive loads are applied. Due to the PM procedure, a fine microstructure is developed. This fine microstructure implies a high strength even for the unreinforced alloy (see figure 2 in ref. 8). Insufficient data concerning the evolution of the RS in the unreinforced alloy (above $2 \%$ strain), however, did not allow determining whether the presence of the reinforcing particles is important for the increase in RS with increased deformation.

The motivation of this investigation is, therefore, to study the generation of RS in aluminum alloys with extensive uniaxial plastic deformation in compressive and tensile modes. For this purpose, two alloys, 2014Al and 6061 Al, were selected. They have high and moderated strength, respectively. In addition, the investigation of the matrix RS evolution with deformation in corresponding MMCs reinforced with $15 \mathrm{vol} \%$ of alumina $\left(\mathrm{Al}_{2} \mathrm{O}_{3}\right)$ particles was also undertaken. 
The present study has, in summary, a threefold objective.

a) The investigation of the influence of alloy strength on the matrix RS evolution with deformation.

b) The evaluation of the reinforcing particles effect (15 vol\% of $\mathrm{Al}_{2} \mathrm{O}_{3}$ ) on the RS evolution.

c) The assessment of the influence of the loading mode (compressive vs. tensile) on the plastic deformation induced RS.

\section{Materials and experiment}

The materials studied in this work were 2014Al (W2A00A), 2014Al-15vol\% $\mathrm{Al}_{2} \mathrm{O}_{3}$ (W2A15A), 6061Al (W6A00A), and 6061Al-15vol\% $\mathrm{Al}_{2} \mathrm{O}_{3}$ (W6A15A). All materials were prepared by ingot metallurgy, IM, and hot extrusion (QED Extrusion Developments Inc.). Conventional metallography was used to reveal the microstructure and to determine the grain size in each material, Table I.

Texture has been determined by X-Ray diffraction at CENIM-CSIC, Madrid, Spain, on polished surfaces of materials in a Siemens Kristalloflex D5000 diffractometer equipped with an open Eulerian cradle. The Schulz reflection method has been used. Pole figures of the 111, 200, 220 and 311 reflections were determined. Details of this technique are found elsewhere [9]. The data have been treated with TexTools software [10] to obtain the orientation distribution functions (ODFs) and, from them, the inverse pole figures for the extrusion axis.

Two sets of samples were machined from each material, one for compressive and one for tensile tests. In each set, several strain levels were applied to different samples up to the highest deformation (i.e., up to $\approx 15 \%$ in compressive deformation and up to fracture in tensile deformation). The compressive samples were cylinders of $6.5 \mathrm{~mm}$ diameter and 13 $\mathrm{mm}$ length. The tensile samples were also cylindrical, $3 \mathrm{~mm}$ diameter and $10 \mathrm{~mm}$ in the gage length, and with threaded heads. Samples were machined with the cylinder axis (loading direction) parallel to the extrusion axis direction. Mechanical tests were carried out at CENIM-C.S.I.C. in a computer controlled SERVOSIS (class 1) testing machine at an initial strain rate of $10^{-4} \mathrm{~s}^{-1}$. Compressive and tensile samples were heat treated to achieve the T6 condition according to the following scheme: solution treatment at $\approx 530^{\circ} \mathrm{C}$ for $90 \mathrm{~min}$, quench in cold water, and ageing at $160^{\circ} \mathrm{C}$ the $2014 \mathrm{Al}$ based materials and at $175^{\circ} \mathrm{C}$ the 6061Al based materials. Different ageing times were used, spanning from 10 to $18 \mathrm{~h}$ depending on the material [11]. For appropriate comparison of uni-axial tensile and 
compressive test data, logarithmic strain, $\varepsilon$, and true stress, $\sigma$, will be used. These magnitudes are defined as: $\varepsilon=\ln (1+e)$ and $\sigma=\frac{P}{A_{0}}(1+e)$, where $P$ is the externally applied force, $A_{0}$ the initial cross-sectional area and $e$ the engineering strain, which is equal to: $e=\frac{\Delta L}{L_{0}}$ (where $\Delta L$ is the increment in length and $L_{0}$ the initial gage length).

For the ex-situ RS measurements, strain scanning by neutron diffraction, ND, of the compressive samples was conducted on D1A diffractometer, at the ILL, Grenoble, France. A neutron wavelength of $1.91 \AA$, and a gage volume of $3 \times 4 \times 1 \mathrm{~mm}^{3}$ were used. Tensile samples were measured by synchrotron radiation diffraction, SRD, on the beam line EDDI at BESSY, Berlin, Germany, which operates in energy dispersive mode, in the range 10 to $150 \mathrm{keV}$. The incoming beam was defined by slits of $100 \mu \mathrm{m}$ height and $1 \mathrm{~mm}$ width, while the diffracted beam size was adjusted by a slit of $30 \mu \mathrm{m}$ height. The grain size of the 2014Al based materials and the W6A15A alloy, Table I, was sufficiently small to have enough diffracted signal for the resulting gage volume used in SRD experiments. The peak position of some reference samples was also measured in order to obtain a suitable unstressed lattice spacing value, $d_{0}$. For the ND measurements, the references were shavings of 2014Al and 6061 Al machined out from the unreinforced alloys. For the SRD measurements, commercial powders of these two alloys were used. The experimental set up for the ND and for the SRD measurements are shown in Figures 1a) and 1b), respectively.

An analysis of the peak width and its evolution with deformation was also conducted. The diffraction peaks were fitted with simple Gaussian functions. Although the instrumental contribution could not be separated, it can be assumed to be the same within every ND and every SRD experiment. Therefore, a peak deconvolution was not needed. In this way, the variations of the peak width on the ND and SRD experiments could be attributed only to microstructural changes with plastic deformation (lattice microscopic strains or type-III mRS [3]) in the unreinforced alloy and the composite matrix.

\section{Residual stress measurement and analysis}

The RS measurement by diffraction techniques is based on the determination of the lattice spacing, $d$, of a given crystallographic ( $h k l)$ by means of Bragg's equation. By comparing the peak position for different spatial directions with that of the unstressed sample, the strain tensor can be calculated. The RS can be then readily determined by applying the generalized 
Hooke's law. This analytical procedure has been explained in detail elsewhere [12]. In both, ND and SRD measurements, the RS analysis has been made on the basis of the Al-311 peak position. This peak shows a relatively high diffracted intensity at any sample orientation despite the strong texture developed in some samples [13]. Furthermore, these grains are elastically and plastically isotropic, i.e. the stress-strain curve follows closely the macroscopic stress-strain behaviour of the alloy. A scattering angle, $2 \theta$ (with $\theta$ the Bragg's angle), of about $90^{\circ}$ for the wavelength used in ND [14] was then found. For both techniques, ND and SRD, the so called $\sin ^{2} \psi$ method has been used [12], where $\psi$ is the angle formed by the extrusion axis and the scattering vector. The measurements were done at the centre of the samples, where the axial and the radial components were obtained. Due to the axial symmetry about the extrusion direction, hoop and radial components are equal at this point.

In the ND experiments, only a narrow range of $2 \theta$ was recorded and only one reflection was measured at a time, since a position sensitive detector was used. The gage volume in ND is large compared to that in SRD. Therefore, the sample has to be large enough to obtain a meaningful signal from a particular region inside the bulk. Consequently, compression samples have been measured with ND. SRD allows measuring smaller samples, or different points inside a sample, thus yielding a higher spatial resolution. Therefore, the $3 \mathrm{~mm}$ diameter tensile samples could be measured on EDDI.

In the SRD experiments, many reflections are obtained at a given $2 \theta$ on using energy dispersive mode. The $(h k l)$ lattice spacing values are calculated from the energy also from Bragg's equation expressing the wavelength in terms of the energy, $E$,

$$
d=\frac{h c}{2 E \sin \theta}
$$

Where $h$ is Planck's constant and $c$ the speed of light. The angle $2 \theta$ was adjusted to $6^{\circ}$. From the $d$ values, the strains were calculated as reported in [12].

\section{Results and Discussion}

The texture of the composites and the unreinforced alloys is shown in the inverse stereographic triangles of Figure 2. A typical $<111>+<100>$ fiber texture (with the fiber axis parallel to the extrusion direction) is developed in the 2014Al based materials. The texture of the alloy is more accentuated than that of the composite; maximum intensities are 25.2 and 2.6, respectively. This difference indicates that the composite matrix has more grains 
randomly oriented revealing a more severe process of particle stimulated nucleation, PSN, for recrystallization, as suggested in [15]. The texture of the 6061 Al based materials is rather weak: $\mathrm{a}<311>$ texture component is developed in the alloy and a $<100>$ in the composite. Their intensities are similar: 2.8 in the alloy and 3.2 in the composite. The texture of these materials is rather unusual, in particular that of W6A00A. The reason for this difference is not yet well known. It should be emphasizes the relatively weak texture of the latter materials indicating that complex recrystallization phenomena should have occurred during extrusion. Further work is presently under progress to investigate further this point.

The mechanical behavior of the four materials is shown in the compressive stress-strain curves of Figure 3. The 2014Al based materials develop, as it is well known, a noticeably higher strength than the $6061 \mathrm{Al}$ based materials. Furthermore, the W2A15A composite reveals a clear higher compressive strength than the $\mathrm{W} 2 \mathrm{~A} 00 \mathrm{~A}$ alloy. This effect is not so obvious in the other composite. This accounts for a more effective load bearing capacity of the reinforcement for the case of the W2A15A composite than for the W6A15A. It should be mentioned, however, that the difference between the W2A15A composite and the W2A00A alloy cannot be only attributed to the presence of the reinforcement. In fact, some difference between the compressive and tensile behavior, a strength differential effect, SDE, in the W2A00A alloy was also observed. Whereas the SDE in composites has been attributed to the presence of a m-RS [16], this effect in the unreinforced alloy is not yet fully understood. Most probably, the texture creates an effect of plastic anisotropy: the grains yielding at lower stress create a residual strain between grains belonging to different texture component (with different $\langle h k l>$ ) upon plastic deformation. This implies that the grains oriented in some directions (in this case the $<111>$ ) act as hard particles [17], thus creating SDE.

Also, the significant larger grain size in the transverse section of the W6A00A alloy than that of the W6A15A composite, Table I, but their similar plastic flow behavior, indicates the irrelevance of the Hall-Petch mechanism in their strengthening. Rather, an interaction mechanism between dislocations and semi-coherent precipitates should govern the strength for plastic flow in these materials. A more complete study of the mechanical behavior and its correlation with microstructure is being the subject of a separate publication.

The evolution of the hydrostatic and axial-deviatoric matrix RS with compressive plastic deformation, as calculated in $[8,16]$, is shown for all materials in the plots of Figure 4 . Due to uncertainties in the measurement of the lattice spacing in the unstressed state, the shavings references did not prove to be fully reliable. For example, their diffraction peak width was 
always higher than that of the bulk alloy, indicating that a large amount of plastic deformation has occurred during shavings machining. Consequently, the relative values of hydrostatic RS, $\Delta \sigma$, have been taken into account: The minimum value of the RS has been taken as an "unstressed" state. A rapid drop of the hydrostatic RS with the onset of plastic deformation results in all materials, Figure 4. The rate of RS decrease is virtually independent of the presence of the alumina particles and slightly higher in the moderate strength 6061Al based materials. These findings lead to the following two important conclusions: a) The ceramic particles play no paramount role in the RS relaxation process with uniaxial compressive plastic strain and b) The softer the matrix alloy the more rapid RS relaxation with the onset of plastic flow occurs. The RS drop is also qualitatively similar to that observed previously in PM 6061Al based alloys and composites [8].

Above some 2\% compressive deformation, a progressive increase of the RS in the 2014Al based materials occurs, Figure 4. This is similar to what it has been reported previously in PM composites [8]. The unreinforced W2A00A alloy also shows the same trend, revealing that this phenomenon is independent of the presence of the reinforcing particles. It should be intimately linked to non homogeneous plastic flow, most likely associated to barreling effect during deformation. On the other hand, the 6061Al based materials do not show such a RS increase above $\approx 2 \%$ deformation. This important difference between the $2014 \mathrm{Al}$ and the 6061 Al based materials should be related to the lower stress required to deform plastically the latter matrix. In other words, it is unfeasible to induce matrix RS upon inhomogeneous compressive plastic deformation in a low strength material, as stated in [18].

Contrary to the hydrostatic RS, the axial-deviatoric RS remains almost unchanged with deformation. This result is also in agreement with previous studies [8]. Apart from some data scatter, a slight drift towards higher RS occurs: the average deviatoric RS is about 20-25 $\mathrm{MPa}$ for the W2A00A alloy and 15-20 MPa for the W2A15A composite. This axial-deviatoric RS term is slightly smaller for the 6061 Al based materials: it is about $5 \mathrm{MPa}$ in the W6A00A alloy and $15 \mathrm{MPa}$ in the W6A15A composite. In the case of the 2014Al based materials the average axial-deviatoric RS is higher in the alloy than in the composite. The contrary is observed in the case of the 6061 Al based materials (dotted and solid lines in the plots of the axial-deviatoric RS of Figure 4 are for the unreinforced alloys and the composites, respectively). This indicates that the higher increase of the hydrostatic RS with plastic deformation and the higher axial-deviatoric-RS in the alloy than in the composite are both associated to the stronger texture of the W2A00A alloy. 
The results of the SRD experiments on the evolution of the RS with tensile plastic deformation of the unreinforced W2A00A alloy are shown in Figure 5. As for the compressive tests of Figure 4, the hydrostatic RS values vs. plastic strain in Figure 5 are plotted relative to the minimum value obtained. The W2A00A underwent the highest level of RS increase with compressive plastic deformation, Figure 4. A rapid drop of the hydrostatic RS occurs already with a small amount of tensile plastic deformation. The magnitude of the RS drop is similar to that in compression, within the experimental error bar. The rate of decrease is, however, slightly smaller. Contrarily to the RS evolution in compression, the hydrostatic RS barely increases with further tensile deformation. Only some slight increase after $15 \%$ deformation (sample tested to failure) is appreciated. Although this sample was measured in a region away from the fracture surface, it may be possible that the RS increase is related to non-homogeneous plastic flow associated to necking. It should be noted that no data between 0.013 and 0.12 strain is available, and further tests should be conducted to confirm these conclusions: As for the compressive behavior, an increase in RS between these strain values could happen. For the case of the axial-deviatoric RS, a progressive decrease, towards negative values occurs.

The behavior is very similar for the W6A15A composite tested in tension. This material underwent a RS increase with compressive plastic deformation higher than the W6A00A alloy (see Figure 4). Figure 6 summarizes the evolution of the RS with tensile plastic deformation in W6A15A. It also shows the decrease of the hydrostatic RS with deformation. No increase of the RS on the sample tested to failure occurs, most probably due to its limited necking. In agreement with the result shown above, Figure 5, tensile deformation leads to a slightly slower RS relaxation than compressive deformation. Little variation of the axialdeviatoric RS occurs. Again, as for the W2A00A alloy, this stress decreases slightly towards negative values with tensile deformation.

To further investigate the evolution of the RS with plastic deformation, the Al-311 peak broadening has been also analyzed. The evolution of the peak width with compressive plastic deformation of all materials investigated is summarized in the plots of Figure 7. Figure 7a) refers to the $2014 \mathrm{Al}$ based materials whereas Figure $7 \mathrm{~b}$ ) to the $6061 \mathrm{Al}$ based materials. Several points can be deduced from these two plots:

1.- A progressive peak broadening with compressive strain occurs in all materials. Broadening is clearly more accentuated in the W2A15A composite than in the W2A00A 
alloy, consistently with the higher strength of the former and, hence, with a higher dislocation density.

2.- Peak broadening of the $6061 \mathrm{Al}$ based materials is very similar in all cases, also in accordance with the similar strength of the composite and the unreinforced alloy in this case.

3.- Peak broadening with compressive strain in the W2A15A composite is more accentuated than in the W6A15A composite. Again, this is in agreement with the higher strength of the former.

The evolution of peak broadening with tensile plastic strain in W2A00A alloy and W6A15A composite is shown in Figure 8. As can be seen, and in agreement with the compressive behavior, peak broadening occurs in both the alloy and the composite with tensile deformation. In this case, however, a large experimental error has been observed for the W2A00A alloy. This does not allow any detailed analysis of FWHM evolution with deformation. This large error is probably due to the large grain size in this alloy, Table I: few grains are covered by the gage volume and the statistics becomes poor.

In conclusion, a rapid RS relaxation occurs, both in compressive and tensile deformation of unreinforced aluminum alloys and the corresponding discontinuously reinforced composites with small strains (i.e., homogeneous deformation). This result, already observed in previous works $[4,5]$, is counterintuitive to simple mechanical models. In fact, the m-RS evolution with plastic deformation is expected to differ from compressive to tensile deformation [4-6, 19]. Tensile plastic deformation should reduce the misfit between matrix and particles, while compressive plastic deformation should increase it. In other words, the initially positive m$\mathrm{RS}$ in the matrix and negative in the reinforcement (consequence of the difference in the coefficient of thermal expansion, CTE) should be reduced (in absolute value) with tensile deformation and increased with compressive deformation.

The results of this work indicate that the plastic flow behavior taking place during compressive and tensile deformation is rather complex. This behavior should not, therefore, be explained on the basis of macroscopic continuum mechanics models, but rather relying on detailed phenomena associated to microstructural changes with deformation, such as those described by dislocation dynamics models. Dislocation motion which takes place during plastic deformation should occur not only to satisfy the sample shape change imposed by the mechanical test (tensile or compressive deformation), but also to decrease the elastic energy associated to the RS generated during thermo-mechanical processes. 


\section{Summary}

The evolution of the residual stress with compressive and tensile plastic deformation in high and moderate strength aluminum alloys and their corresponding metal matrix composites (reinforced by $15 \%$ vol. alumina particles) has been investigated. The following are the most interesting findings of this research.

a) A rapid drop, of about $120 \mathrm{MPa}$, of the hydrostatic RS occurs with small $(<2 \%)$ plastic uniaxial deformation of the composites and unreinforced alloys. This RS decay is independent of the mode of testing (tensile vs. compressive), and the presence of the reinforcement. The RS decay is also found to be slightly slower the higher the strength of the aluminum alloy matrix. The axial-deviatoric RS, however, undergoes little variation with strain: In essence, it remains constant with strain.

b) At higher strains, compressive deformation leads clearly to a RS increase in the materials with the high matrix strength (2014Al). This increase is independent of the presence of alumina reinforcing particles, but is associated to materials strength: the higher the strength, the higher the RS increase with further strain.

c) Although more experiments are required, no RS increase is evident with tensile deformation. This supports the idea that non-homogeneous deformation processes associated to barreling, are responsible of the RS increase with large compressive deformation. The axial-deviatoric RS decreases slightly in the unreinforced W2A00A alloy and the W6A15A composite.

It can be concluded finally that the re-generation of a RS by non-homogeneous plastic deformation associated to barreling should occurs more likely in high strength materials since the internal stress that these materials undergo during plastic deformation is also high. Further work is needed to understand in depth the ultimate mechanisms that account for the RS evolution reported in this and previous investigations.

\section{Acknowledgements}

Project MAT-05-0527 from MEC, Spain. Support from the ILL for the ND measurements on D1A and from BESSY for the SRD experiments on EDDI under contract $n^{\circ}$ RII3.CT-2004506008 is also gratefully acknowledge. Special thanks are due to Dr. Crhistoph Genzel and Manuela Klaus for their help in the SRD measurements on EDDI. 


\section{References}

[1] T.W. Clyne, P.J. Withers: An introduction to metal matrix composites, Cambridge Univ. Press, UK, 1993.

[2] M. Taya, R.J. Arsenault: Metal matrix composites Pergamon Press, Oxford, UK, 1989.

[3] L. Pintschovius, in: M.T. Hutchings, A.D. Krawitz (Eds.), Proceedings of the NATO Advanced Research Workshop on Measurements of Residual and Applied Stress Using Neutron Diffraction, (Kluwer Academic Publishers, Dordrecht, 1992, pp.115-130.

[4] M.E. Fitzpatrick, P.J. Withers, A. Baczmanski, M.T. Hutchings, R. Levy, M. Ceretti, A. Lodini, Acta Mater. 50 (2002) 1031-1040.

[5] R. Levi-Tubiana, A. Baczmanski, A. Lodini, Mat. Sci. Eng. A341 (2003) 74-86.

[6] M.E. Fitzpatrick, M. Dutta, L. Edwards, Mater. Sci. Technol. 14 (1998) 980-986.

[7] M.E. Fitzpatrick, M.T. Hutchings, P.S. Withers, Acta Mater. 47 (1999) 585-593.

[8] R. Fernández, G. Bruno, G. González-Doncel, Mat. Sci. Eng. A403 (2005) 260-268.

[9] M.T. Pérez-Prado, M.C. Cristina, O. Ruano, G. González-Doncel, Mat. Sci. Eng. A244 (1998) 216-223.

[10] http://www.resmat.com/

[11] ASM Handbook, Vol. 4 “Heat Treating”. ASM International 1991, p 841-879.

[12] V. Hauk: Structural and residual stress analysis by non destructive methods: Evaluation- Application- Assessment, Elsevier, Amsterdam, 1997.

[13] R. Fernández, G. González-Doncel, G. Bruno, in ICCM-15. ed. by V.E. Verijenko, S. Adali, E. Morozov, C.J. von Klemerer. ISBN: 1-86840-589-3, Durban, South Africa, June 2005.

[14] G. Bruno, R. Fernández, G. González-Doncel, Mat. Sci. Eng. A382 (2004) 188-197.

[15] A. Borrego, R. Fernández, M.C. Cristina, J. Ibañez, G. González-Doncel, Comp. Sci. Technol. 62 (2002) 731-742.

[16] R. Fernández, G. Bruno, G. González-Doncel, Acta Mater. 52 (2004) 5471-5483.

[17] G. González-Doncel, M. Torralba, O. Ruano, Acta Metall Mater. 39 (1991) 2393-2403.

[18] I. Dutta, J.D. Sims, D.M. Seigenthaler, Acta Metall Mater. 41 (1993) 885-908.

[19] A.M.Korsunsky, M.R.Daymond, K.E.James, Mat. Sci. Eng. A334 (2002) 41-48. 


\begin{tabular}{|l|c|c|}
\hline Material & $\begin{array}{c}\text { Grain Size }(\boldsymbol{\mu m}) \\
\text { Transverse section }\end{array}$ & $\begin{array}{c}\text { Grain Size }(\boldsymbol{\mu}) \\
\text { Longitudinal section }\end{array}$ \\
\hline W2A00A & $24 \pm 10$ & - \\
\hline W2A15A & $12 \pm 6$ & $18 \pm 12$ \\
\hline W6A00A & $132 \pm 66$ & - \\
\hline W6A15A & $17 \pm 6$ & $40 \pm 16$ \\
\hline
\end{tabular}

Table I. - Grain size values of materials. 


\section{Figure captions}

Figure 1.- Experimental set-up for the RS measurements on a) D1A (at the ILL, Grenoble, France), for the compressive samples and b) EDDI (at BESSY, Berlin, Germany) for the tensile samples. The inset in each figure is a detail of the samples measured.

Figure 2.- Inverse pole figures of the extrusion axis showing the texture of the unreinforced alloys and the Al alloy phase of the composites.

Figure 3.- Compressive behavior (true stress vs. logarithmic strain) in T6 condition of the 2014Al (W2A00A) and 6061Al (W6A00A) alloys and the corresponding composites investigated.

Figure 4.- Evolution of the total residual stress (hydrostatic and axial-deviatoric terms) with compressive plastic deformation of the 2014Al (W2A00A) and 6061Al (W6A00A) alloys and the corresponding composites. Note that relative hydrostatic and absolute axialdeviatoric residual stress values are represented.

Figure 5.- Evolution of the residual stress (hydrostatic and axial-deviatoric terms) with tensile plastic deformation of the unreinforced W2A00A alloy. Note that relative hydrostatic and absolute axial-deviatoric residual stress values are represented on the same scale. Error bars are contained in the markers (typically 5-6 MPa)

Figure 6.- Evolution of the matrix residual stress (hydrostatic and axial-deviatoric terms) with tensile plastic deformation of the W6A15A composite. As in previous figure, relative hydrostatic and absolute axial-deviatoric residual stress values are represented on the same scale. Error bars are contained in the markers (typically 5-6 MPa)

Figure 7.- Evolution of the FWHM of the Al-(311) peak with compressive plastic strain in, a) the $2014 \mathrm{Al}$ based materials and b) the 6061 Al based materials. Error bars are contained in the markers (typically $<0.005^{\circ}$ )

Figure 8.- Evolution of the Al-(311) FWHM with tensile plastic strain in the W2A00A alloy and W6A15A composite. 

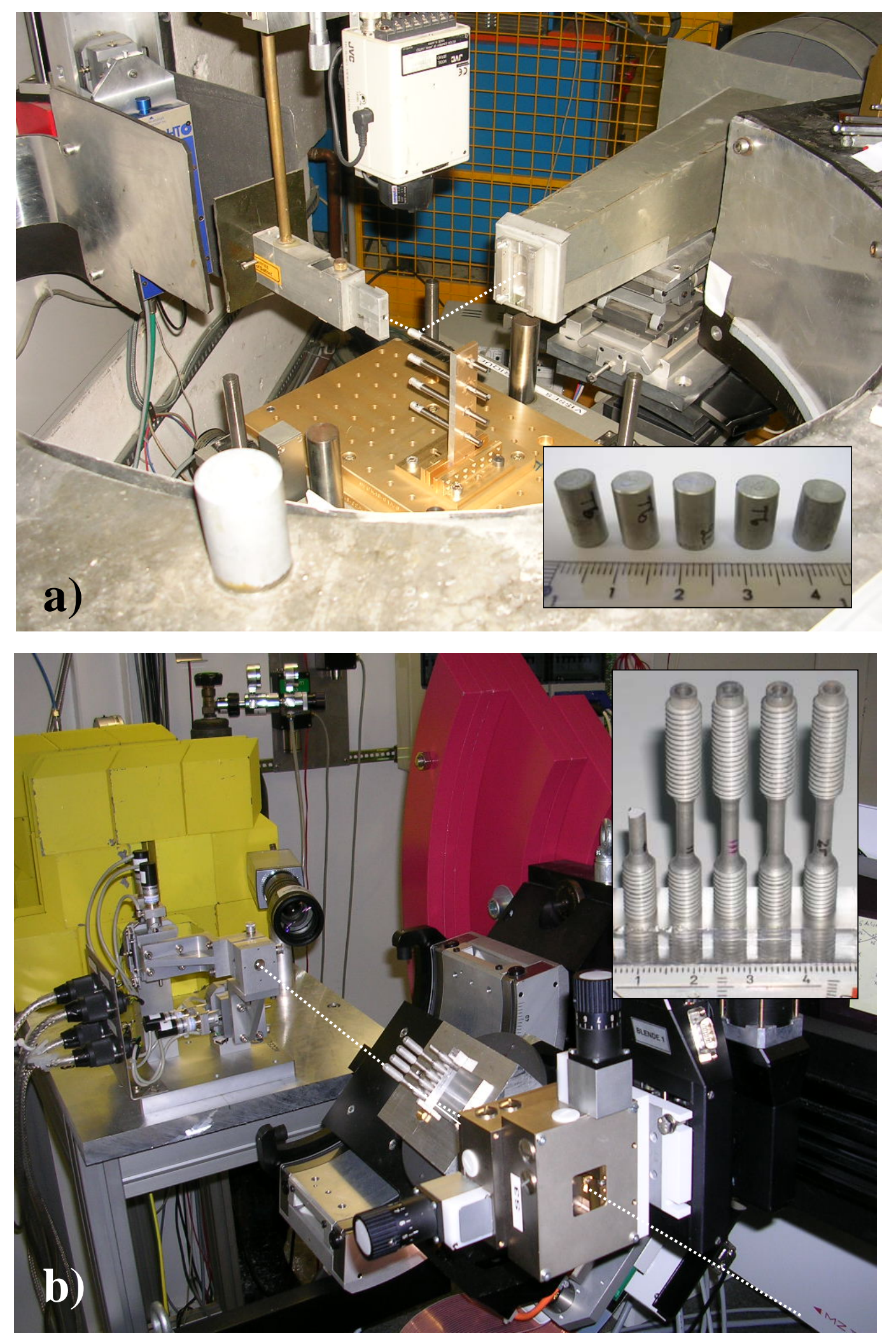

Figure 1.- Experimental set-up for the RS measurements on a) D1A (at the ILL, Grenoble, France), for the compressive samples and b) EDDI (at BESSY, Berlin, Germany) for the tensile samples. The inset in each figure is a detail of the samples measured. 

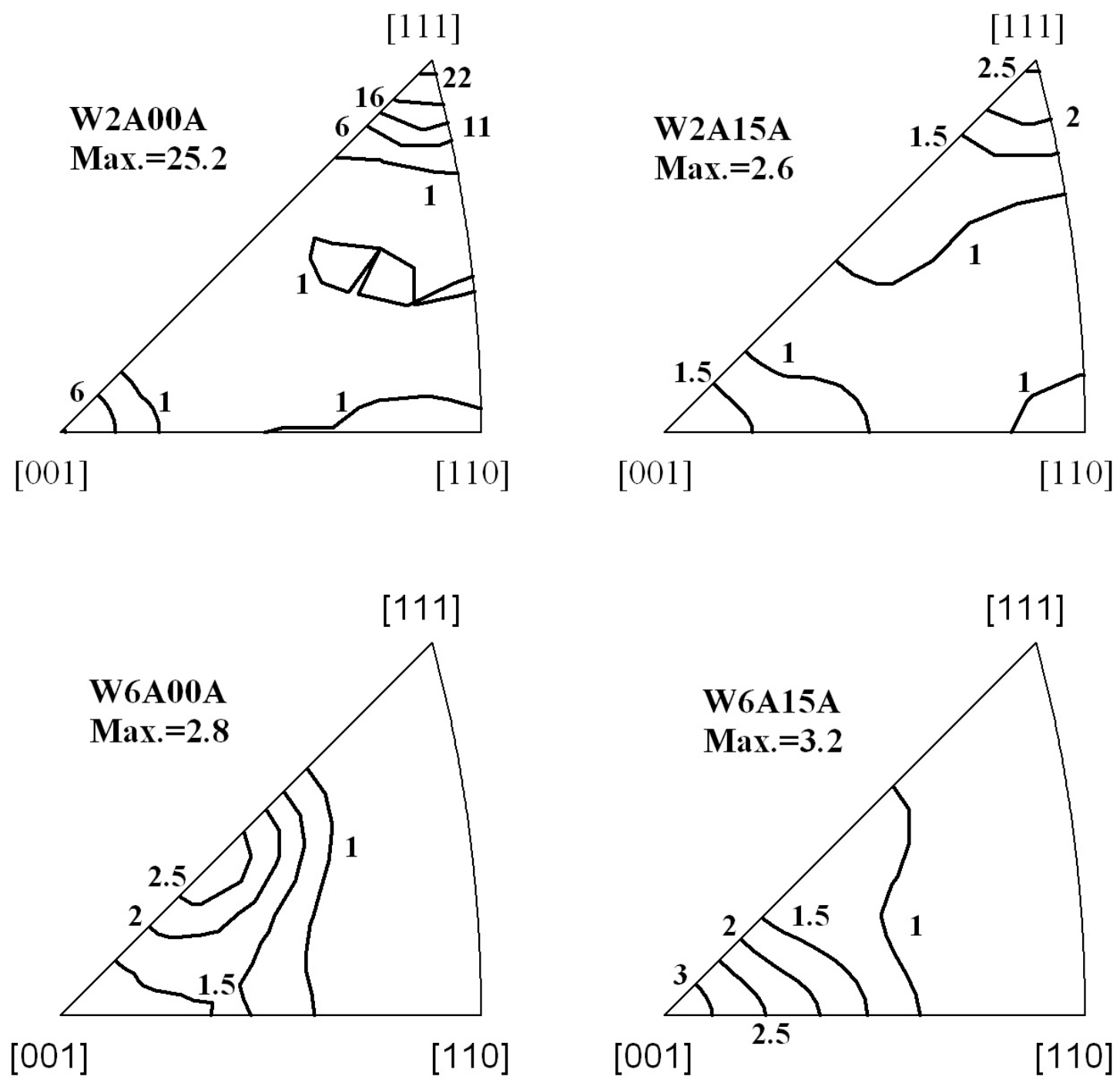

Figure 2.- Inverse pole figures of the extrusion axis showing the texture of the unreinforced alloys and the $\mathrm{Al}$ alloy phase of the composites. 


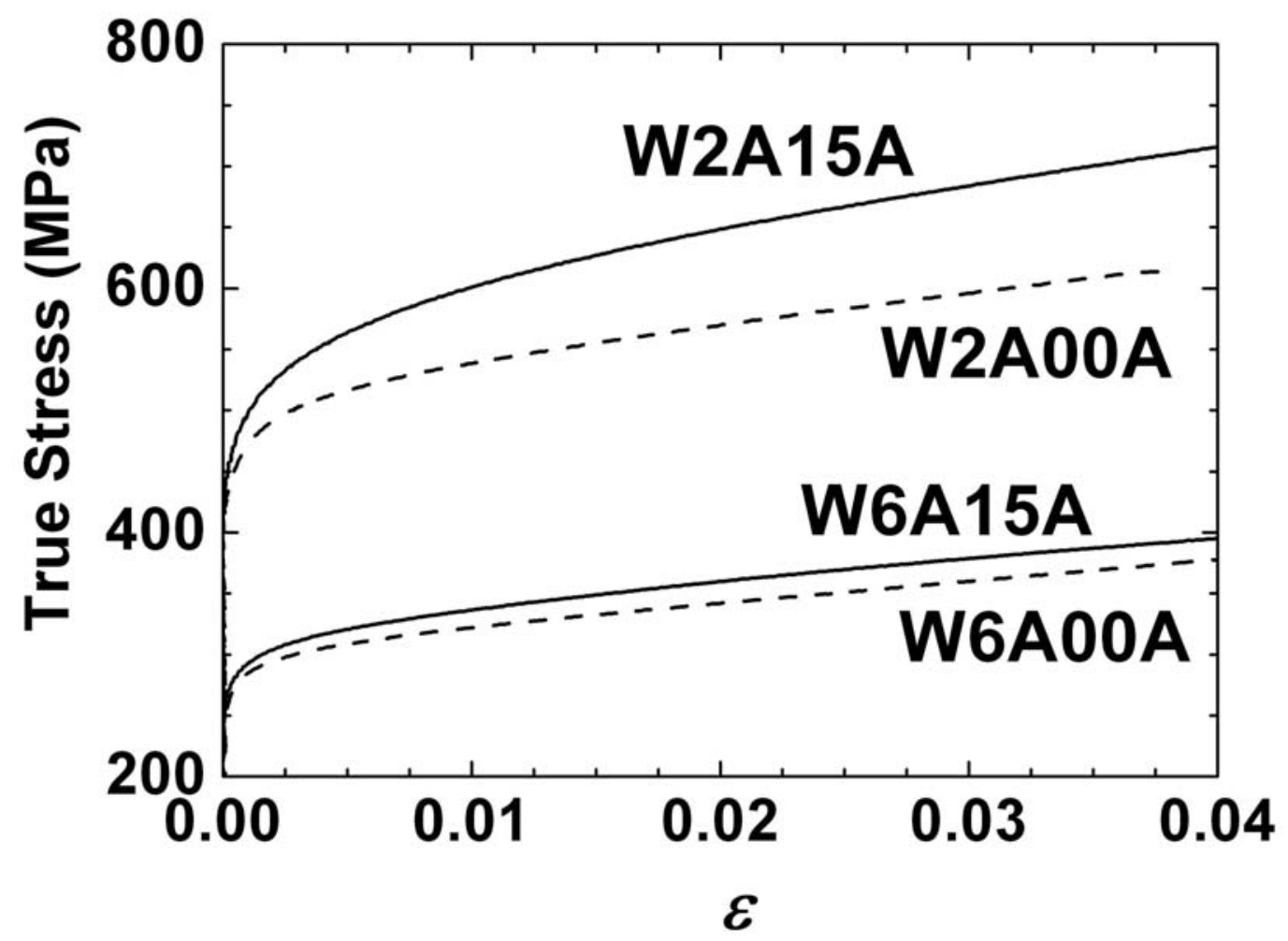

Figure 3.- Compressive behavior (true stress vs. logarithmic strain) in T6 condition of the 2014Al (W2A00A) and 6061Al (W6A00A) alloys and the corresponding composites investigated. 

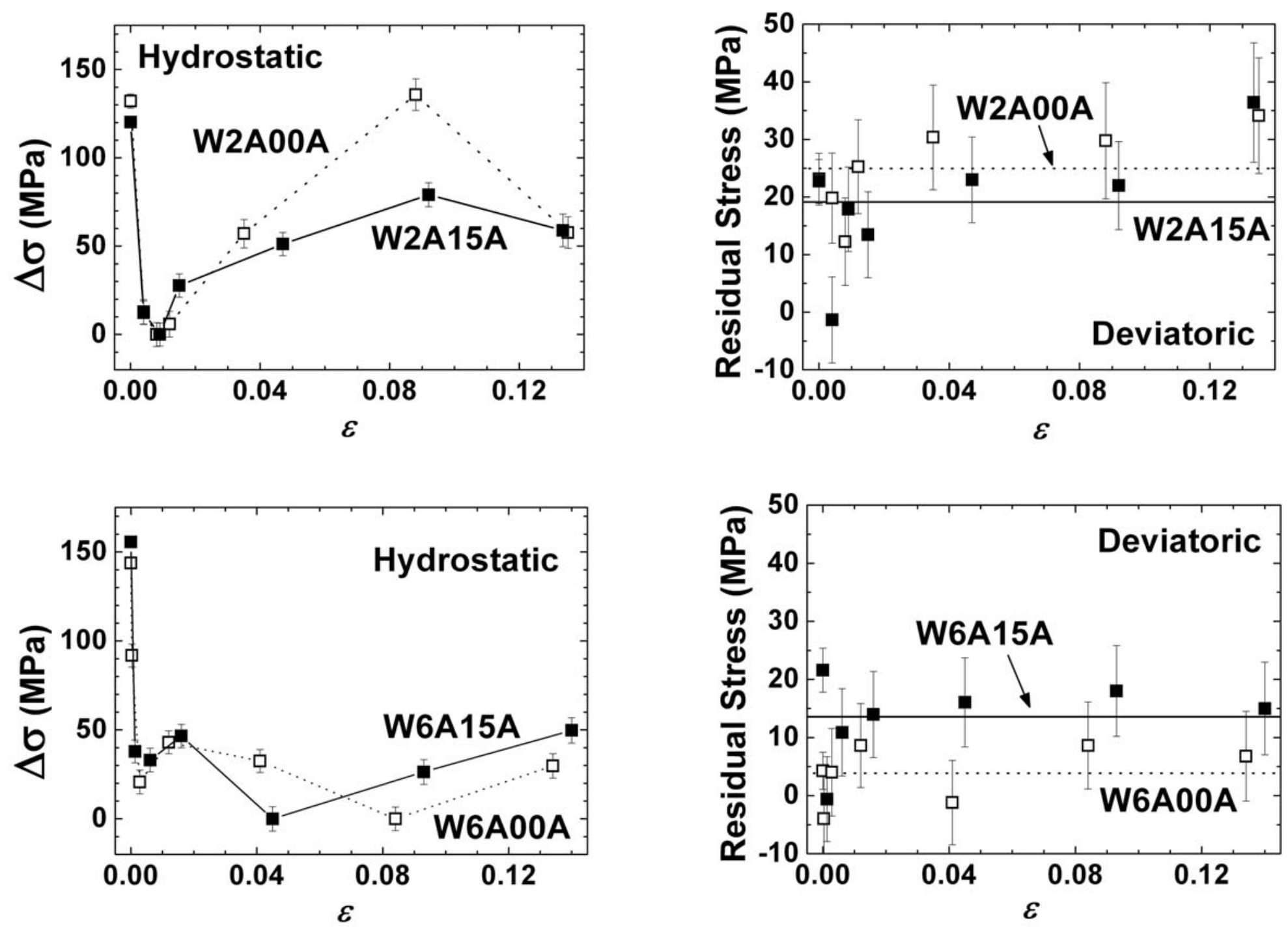

Figure 4.- Evolution of the total residual stress (hydrostatic and axial-deviatoric terms) with compressive plastic deformation of the 2014Al (W2A00A) and 6061 Al (W6A00A) alloys and the corresponding composites. Note that relative hydrostatic and absolute axial-deviatoric residual stress values are represented. 


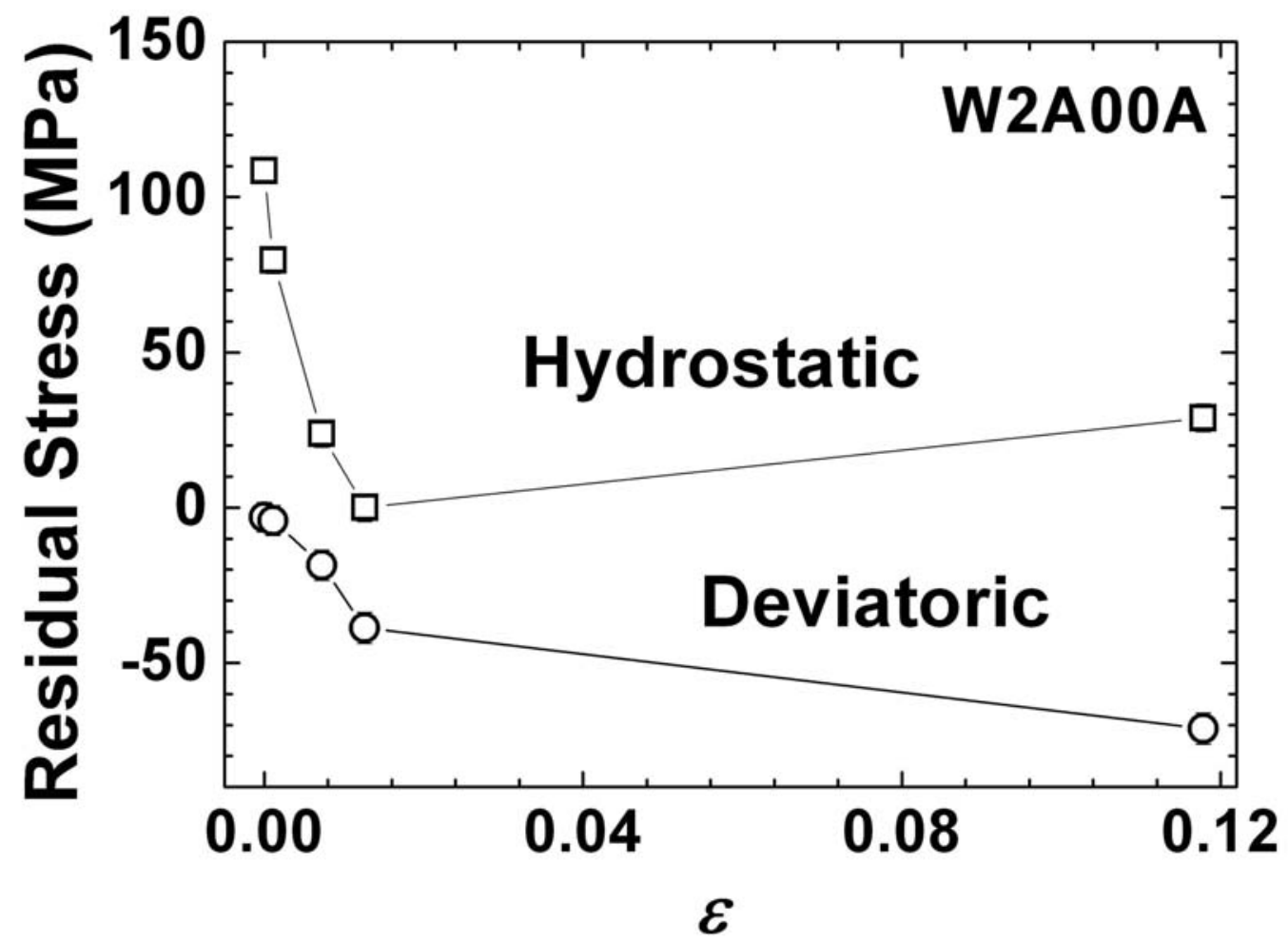

Figure 5.- Evolution of the residual stress (hydrostatic and axial-deviatoric terms) with tensile plastic deformation of the unreinforced W2A00A alloy. Note that relative hydrostatic and absolute axial-deviatoric residual stress values are represented on the same scale. Error bars are contained in the markers (typically 5-6 MPa) 


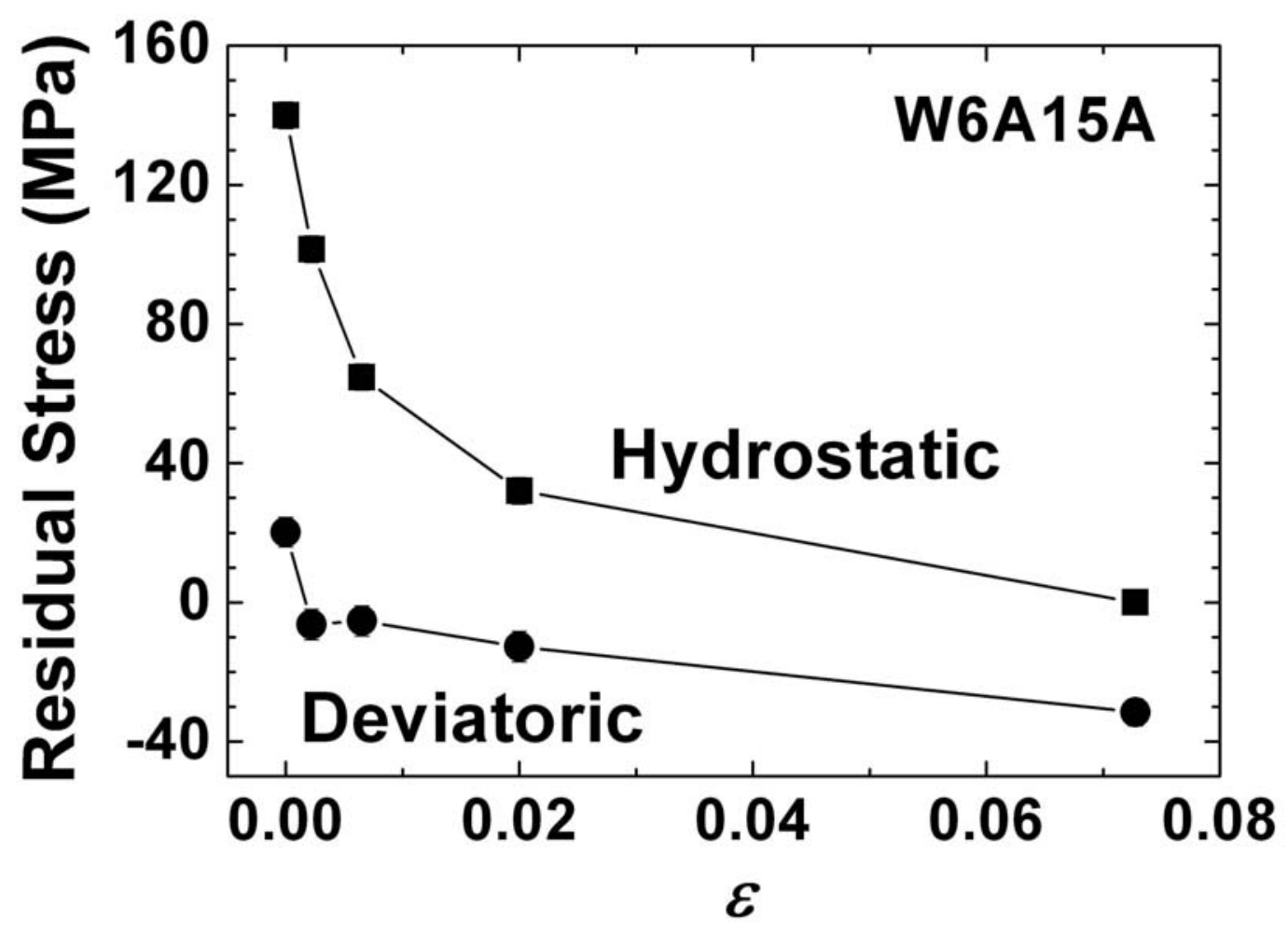

Figure 6.- Evolution of the matrix residual stress (hydrostatic and axial-deviatoric terms) with tensile plastic deformation of the W6A15A composite. As in previous figure, relative hydrostatic and absolute axial-deviatoric residual stress values are represented on the same scale. Error bars are contained in the markers (typically 5-6 MPa). 

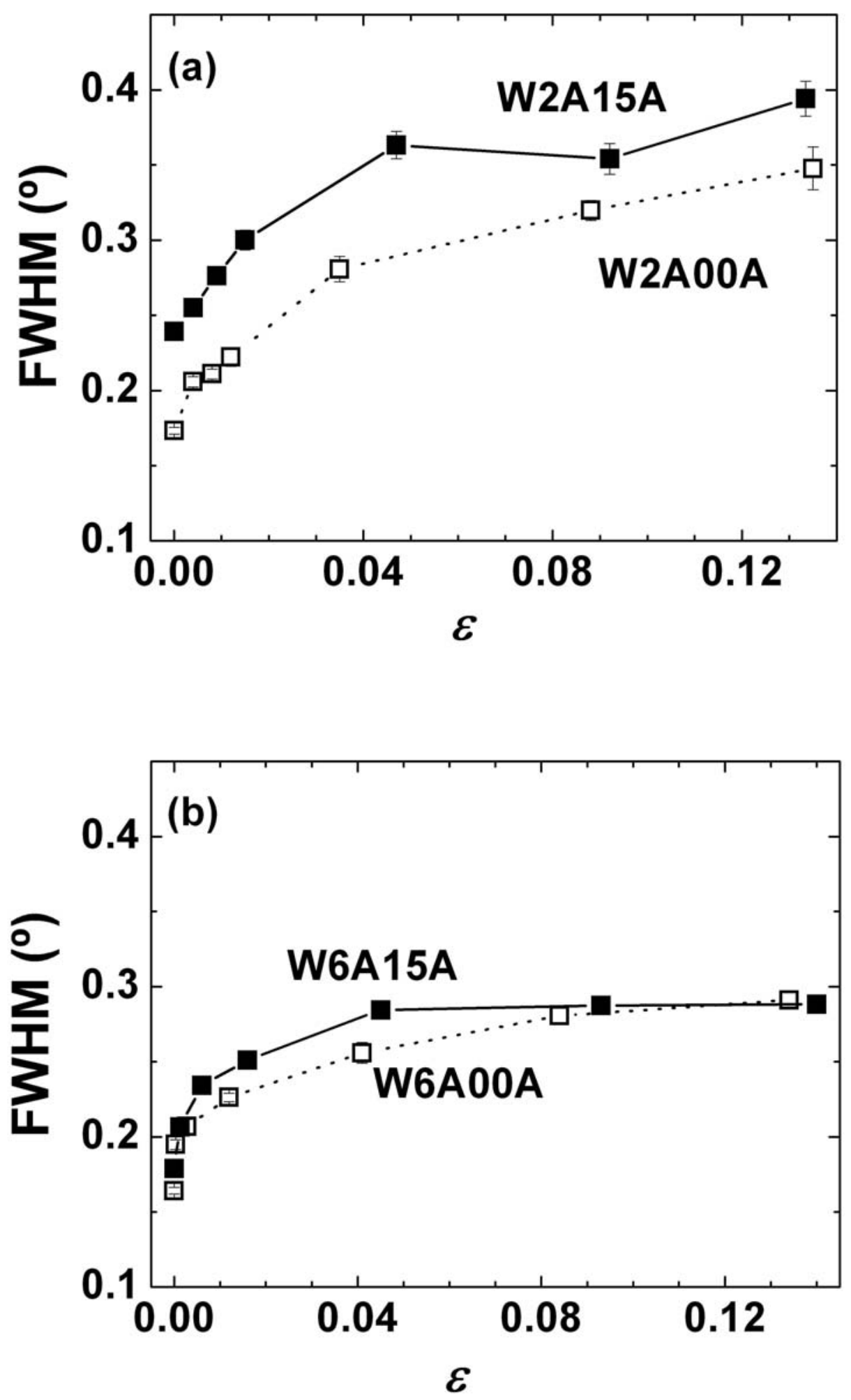

Figure 7.- Evolution of the FWHM of the Al-(311) peak with compressive plastic strain in, a) the 2014Al based materials and b) the 6061Al based materials. Error bars are contained in the markers (typically $<0.005^{\circ}$ ). 


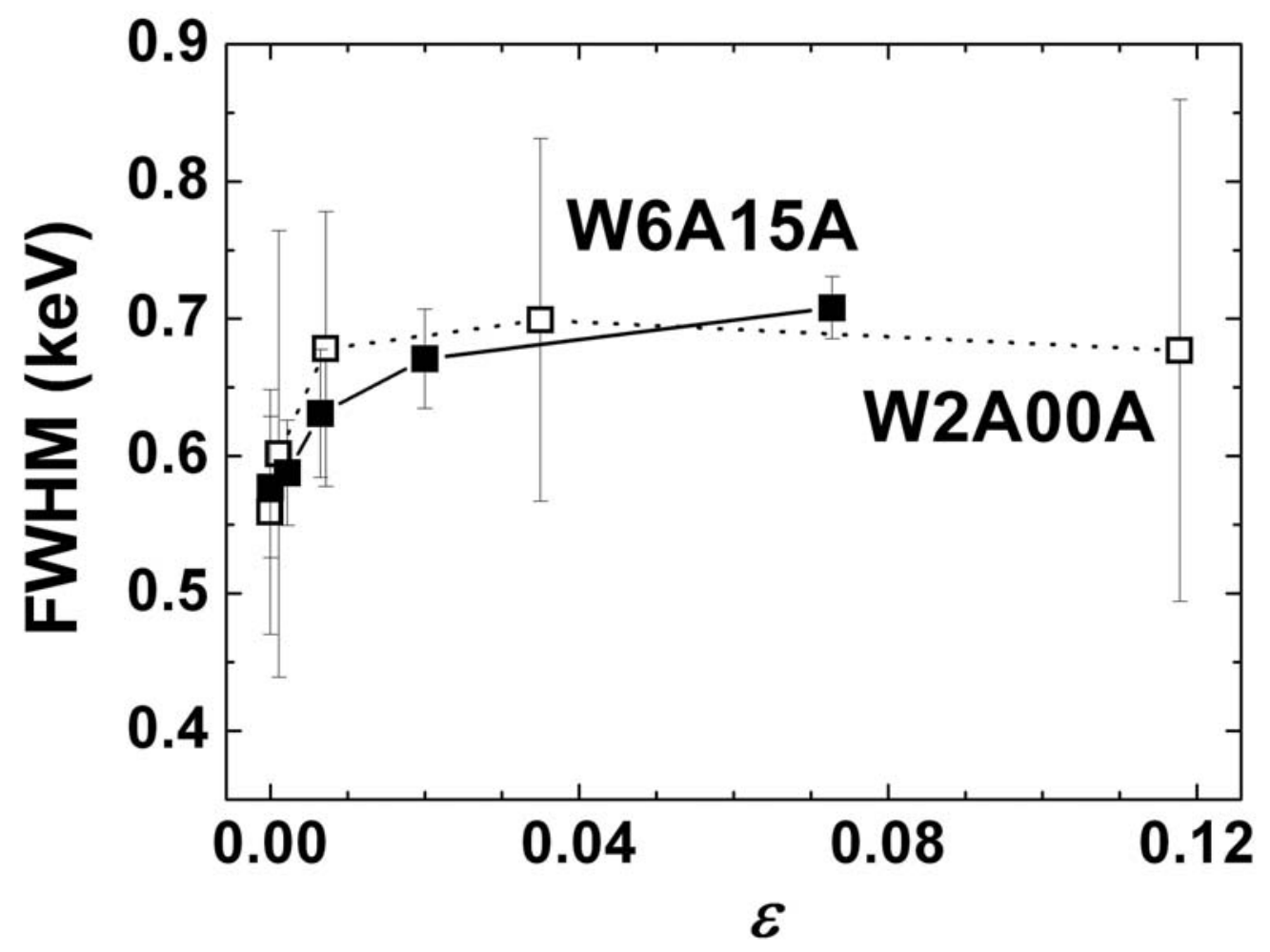

Figure 8.- Evolution of the Al-(311) FWHM with tensile plastic strain in the W2A00A alloy and W6A15A composite. 